raised questions that were addressed by various speakers. R. Sancisi (Groningen Univ.) said that some of the nearby galaxies that he had studied using the 21-cm-wavelength (neutral-hydrogen) line are very extended, so even nearby galaxies may be larger on average than has been believed. But there is considerable uncertainty in comparing $21-\mathrm{cm}$ radio data with optical (or ultraviolet) data, mainly because the radio technique can detect neutral hydrogen only at densities 10,000 times larger than those routinely detected optically, simply because of the difference in line-strength. F. Briggs (Pittsburgh Univ.) showed how the detailed velocity structure and the spatial extent of the strongest hydrogen absorbers can be inferred using $21-\mathrm{cm}$ absorption against a quasar.

Even in the early Universe, the abundances of heavy elements were not very different from those found in the interstellar medium in our Galaxy today, which indicates that early nucleosynthesis and enrichment of the interstellar medium by massive stars probably occurred (J. Bergeron, Univ. Paris; C. Blades, Space Telescope Science Institute). The systems seen all have similar velocity structures as might be expected if they are in galaxies (W. Sargent, Caltech). A major difference between the highredshift material and the galactic interstellar medium is found in the $\mathrm{H}_{2} / \mathrm{H}$ ratio, which has a value near unity in many regins in our Galaxy. Quasar 0528-250, one of two which were investigated, reveals a system with redshift $z \sim 2.8$ in which $\mathrm{H}, / \mathrm{H}$ is about $10^{-3}$.

Another link with real galaxies was indicated by B. Savage (Wisconsin Univ.) who described the ultraviolet observations of the interstellar medium in our Galaxy. In particular, the key tracers for the hot interstellar medium are highly ionized species such as $\mathrm{N}^{4+}$ and $\mathrm{O}^{5+}$. These are rarely seen in quasar absorption systems, but are common in our Galaxy. This might be a genuine difference, but the situation is often ambiguous. The $\mathrm{N}^{4+}$ and $\mathrm{O}^{5+}$ lines in the quasar spectrum could be swamped by neutral-hydrogen (Lyman- $\alpha$ ) lines with lower redshift than the quasar, and so they could have been missed. In a further comparison with galactic interstellar medium, D. York (Chicago Univ.) highlighted the difficulty of finding low-redshift systems similar to the high-redshift absorbers, and suggested that gas-rich systems such as galaxies undergoing bursts of star formation could be appropriate candidates.

The hydrogen lines themselves were also discussed. Although an individual quasar spectrum will generally reveal only a few systems containing heavy elements, there are invariably many - several hundred in the highest-redshift cases - absorbing regions in which only hydrogen is

\title{
A case of mistaken identity in swordtail fish
}

WHY do members of different species avoid mating with each other? The classical explanation is that hybrid inviability results in selection for premating isolating mechanisms $^{1}$. As we have discussed ${ }^{2}$ in a previous News and Views article, an alternative route is sexual selection: mating preferences have an evolutionary dynamics of their own, and can evolve in the absence of contact with other populations or species $^{3,4}$. If a geographical barrier divides a population into two, mating preferences can evolve to differ in the subpopulations. When the barrier is removed, choice of

\section{IMAGE UNAVAILABLE FOR COPYRIGHT REASONS}

that this preference is a consequence of the elaborate courtship display of the male $X$. nigrensis. Unfortunately, the exact basis of the $X$. nigrensis female mating preference remains unknown because Ryan and Wagner did not perform the same analysis using these females.

What do these results mean in evolutionary terms? The females of the two species have very similar mating preferences, but $X$. nigrensis males seem to have responded much more strongly to this sexual selection than $X$. pigmaeus males. This difference between the species suggests that the female preference has not been produced entirely by coevolution with the preferred male character, as is implied in many models of sexual selection $^{3,6,7}$. The mating preference may instead have been present in the common ancestral species, and the male $X$. pigmaeus fail to respond either because they lack

The larger fish is the swordtail $\mathrm{X}$. nigrensis, the smaller is the necessary genetic variX. pigmaeus. (Courtesy of Michael J. Ryan.)

mate alone could prevent hybridization between the two populations. However, a recent study by Ryan and Wagner ${ }^{5}$ of swordtail fish suggests that hybridization between populations can sometimes be favoured by sexual selection: females of one 'species' actually prefer to mate with males of another.

In the swordtail species Xiphophorus nigrensis, only the larger males have an elaborate courtship display and it is those males that are preferred as mates by conspecific females. Sexual selection therefore favours males with some combination of large body size, elaborate courtship, and perhaps a further, undetected character with which both of these are correlated. $X$. pigmaeus males lack the swordtail that is found in all $X$. nigrensis males, but are like small $X$. nigrensis males in both size and courtship behaviour (see figure). Given a visual choice, $X$. pigmaeus females approach all but the smallest $X$. nigrensis males in preference to those of their own species. Subsequent tests demonstrated

detected. Several investigations into the dependence of the number of these latter systems on redshift conclude that they were more numerous in the past. Detailed studies, mainly by R.W. Hunstead (Sydney Univ.) and his collaborators, and by D. Tytler (Columbia Univ.) show that the numbers of detectable clouds decrease as their redshift becomes close to that of the quasar. This is probably because proximity to the bright quasar causes more of the hydrogen to be ionized and, because we can detect only the neutral hydrogen, fewer systems are seen. By extension, J.P. Ostriker (Princeton Univ.) pointed out subject to some opposing selective force (such as high predation rates) on courting males. Sexual selection may thus have made hybridization between the two species more likely if their ranges come to overlap. The mating preference of the $X$. pigmaeus females for most classes of $X$. nigrensis males would result in an excess of this type of hybrid mating. Because matings between the species appear to produce viable, fertile offspring, the two species could become one. Linda Partridge \& Paul H. Harvey

1. Dobzhansky, Th. Genetics of the Evolutionary Process (Columbia University Press, New York, 1970).

2. Partridge, L. \& Harvey, P. H. Nature 323, 580-581 (1986)

3. Lande, R. Proc. natn. Acad. Sci. U.S.A. 78, 3721-3725 (1981)

4. Patterson, H.E.H. S. Afr. J. Sci. 78, 53-57 (1982)

5. Ryan, M.J. \& Wagner, W.E. Science 236, 595-597 (1987).

6. O'Donald, P. Genetic Models of Sexual Selection (Cambridge University Press, 1980).

Kirkpatrick, M. Evolution 36, 1-12 (1982).

Linda Partridge is in the Department of Zoology, University of Edinburgh, Edinburgh EH9 3JT, Paul H. Harvey is in the Department of Zoology, University of Oxford, Oxford OX1 3PS, UK.

that if there was a quasar between us and the quasar against which the absorption is seen this would also reduce the numbers of hydrogen lines observed. This possibility remains to be tested.

Another critical quantity of these socalled 'Lyman forest' systems is the velocity spread revealed by the lines. Published estimates suggest that the fullwidth-half-intensity for the velocity distribution is typically $50-60 \mathrm{~km} \mathrm{~s}^{-1}$. If this width is caused solely by thermal motion, then it implies temperatures of about $70,000 \mathrm{~K}$, considerably higher than theories suggest. The most likely explanation 